\section{LUPUS SCIENCE\& MEDICINE}

\title{
Topical drug-induced subacute cutaneous lupus erythematosus isolated to the hands
}

\author{
Sarika M Ramachandran, ${ }^{1}$ Jonathan S Leventhal, ${ }^{1}$ Loren G Franco, ${ }^{1}$ Adnan Mir, ${ }^{1}$ \\ Ruth F Walters, ${ }^{1}$ Andrew G Franks $\mathrm{Jr}^{1,2}$
}

To cite: Ramachandran SM, Leventhal JS, Franco LG, et al. Topical drug-induced subacute cutaneous lupus erythematosus isolated to the hands. Lupus Science \& Medicine 2017;4:e000207. doi:10.1136/lupus-2017000207

Received 17 January 2017 Revised 11 February 2017 Accepted 14 February 2017

\section{CrossMark}

\footnotetext{
${ }^{1}$ The Ronald 0. Perelman Department of Dermatology, New York University School of Medicine, New York, New York, USA

${ }^{2}$ Division of Rheumatology, Department of Medicine, New York University School of Medicine, New York, New York, USA
}

Correspondence to Professor Andrew G Franks, Jr; andrew.franks@nyumc.org

\section{ABSTRACT}

Subacute cutaneous lupus erythematosus (SCLE) is a well-defined subtype of lupus erythematosus, characterised by photosensitivity, annular and/or psoriasiform lesions, variable systemic involvement and presence of circulating SSA/anti-Ro antibodies. SCLE may be idiopathic or drug-induced. Both the idiopathic and drug-induced forms of SCLE are analogous in their clinical, serological and histological features. Drug-induced SCLE has been reported with various oral agents, but to our knowledge this is the first reported case due to a topical medication. A 34-year-old female foot masseuse presented with a 2-month history of scaly, erythematous lesions isolated to the dorsal hands and interdigital spaces. She had used topical terbinafine, a topical antifungal cream, to her clients' feet for a number of years. ANA and anti-SSA/Ro antibodies were positive. Physical examination, serology and histopathology were consistent with SCLE. We propose that our patient's unique presentation of SCLE may be explained by a prolonged occupational exposure to topical terbinafine as a foot masseuse. While oral terbinafine is a drug known to cause drug-induced SCLE, to our knowledge, this is the first topically induced form of the disease.

\section{INTRODUCTION}

Cutaneous lupus erythematosus (CLE) is an autoimmune disease characterised by a wide spectrum of skin lesions that typically occur on sun-exposed areas of the skin. In addition to the classical forms of CLE, which include discoid lupus erythematosus and subacute cutaneous lupus erythematosus (SCLE), there are uncommon subsets and atypical clinical variants, which may be diagnostically challenging. ${ }^{1}$ Furthermore, different forms of cutaneous lupus may be induced by various drugs. Drug-induced SCLE has been associated with common systemic medications such as hydrochlorothiazide, calcium channel blockers, angiotensin-converting enzyme inhibitors, proton pump inhibitors, tumour necrosis factor (TNF)- $\alpha$ inhibitors, chemotherapy agents and terbinafine. ${ }^{1-3}$

We report the case of a foot masseuse with an unusual presentation of SCLE isolated to the dorsum of the hands and interdigital spaces. We propose that occupation as a foot masseuse may have predisposed the patient to sensitisation to topical terbinafine and may explain the patient's condition. The diagnosis, aetiological factors and pathophysiology will be discussed.

\section{CASE REPORT}

An otherwise healthy foot masseuse presented to the Dermatology Clinic at Bellevue Hospital Center with a chief complaint of hand lesions for 2 months, associated with skin peeling and itchiness. The patient had no other medical complaints, no significant medical history and was on no other medications. The patient reported working as a foot masseuse for many years and admitted to regularly applying topical terbinafine to the clients' feet without gloves. The patient denied any history of hand lesions prior to entering this profession.

Physical examination revealed welldemarcated, scaling, erythematous plaques with hyperpigmented borders and some erosive, indurated plaques interdigitally and on the dorsum of the hands (figure 1). The lesions were confined to the dorsum of the hands and interdigital spaces. No similar lesions were found elsewhere on the body. A Potassium hydroxide $(\mathrm{KOH})$ was negative, and the patient's hand dermatitis was treated as a contact dermatitis with clobetasol $0.05 \%$ ointment. On return visit 1 month later, there was no improvement and fungal culture was also negative. Two skin biopsies were then performed on the left fourth digit and left dorsal hand.

Histopathology of both skin biopsies revealed superficial and deep perivascular, periadnexal and band-like infiltrate 


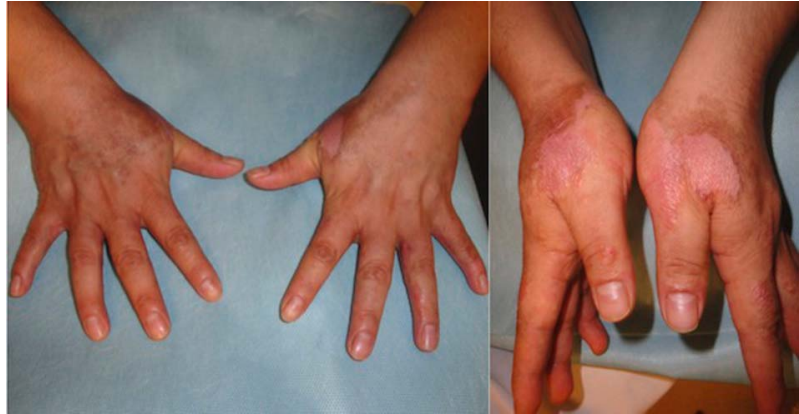

Figure 1 Topical terbinafine-induced subacute cutaneous lupus erythematosus. Scaling erythematous plaques with hyperpigmented borders and erosive, indurated plaques interdigitally.

comprised of lymphocytes, histiocytes and rare plasma cells. Lymphocytes extended to the overlying, slightly hyperplastic epidermis with vacuolar changes, numerous necrotic keratinocytes and hyperkeratosis with foci of parakeratosis. A Periodic acid-Schiff (PAS)-D stain revealed slight thickening of the basement membrane and no evidence of fungal hyphae. A colloidal iron stain revealed increased deposits of connective tissue mucin throughout the dermis, most prominent in the reticular dermis. No spongiosis was noted (figure 2A,B). These findings were most consistent with a connective tissue disease such as discoid lupus or SCLE.

Laboratory evaluation performed after biopsy revealed normal complete blood count, kidney function, hepatic panel and urinalysis. ANA was positive in a speckled pattern at a titre of 1:40, anti-Anti-Sjögren's syndrome type A (SSA)/Ro antibody was positive at 1.6 Antibody Index (AI) and anti-Anti-Sjögren's syndrome type B (SSB)/La antibody was negative. Based on the clinical history, physical examination, histopathology and serology, the diagnosis of acral SCLE was made. After discontinuation of topical terbinafine, the lesions gradually resolved, and on telephone follow-up 6 months later there has been no further recurrence of the illness.

\section{DISCUSSION}

CLE is a highly photosensitive condition that usually affects sun-exposed areas of the face and scalp. ${ }^{1}$ It does not commonly affect the hands in isolation without any other lesions in more photosensitive areas of the body. A literature search disclosed many reports on CLE and involvement of acral surfaces, none of which described this rare and atypical clinical presentation of isolated dorsal and interdigital SCLE. ${ }^{4}$ Of those cases reported in the literature, the majority presented with discoid lesions. ${ }^{45}$ Our patient had primarily scaly, erythematous lesions of the fingers and interdigital spaces that were clinically most consistent with SCLE and supported by positive speckled ANA and positive anti-SSA/Ro antibodies. ${ }^{1267}$ SCLE may be induced by various oral medications, most commonly antihypertensive agents and oral terbinafine. ${ }^{2}$ One study found that patients with
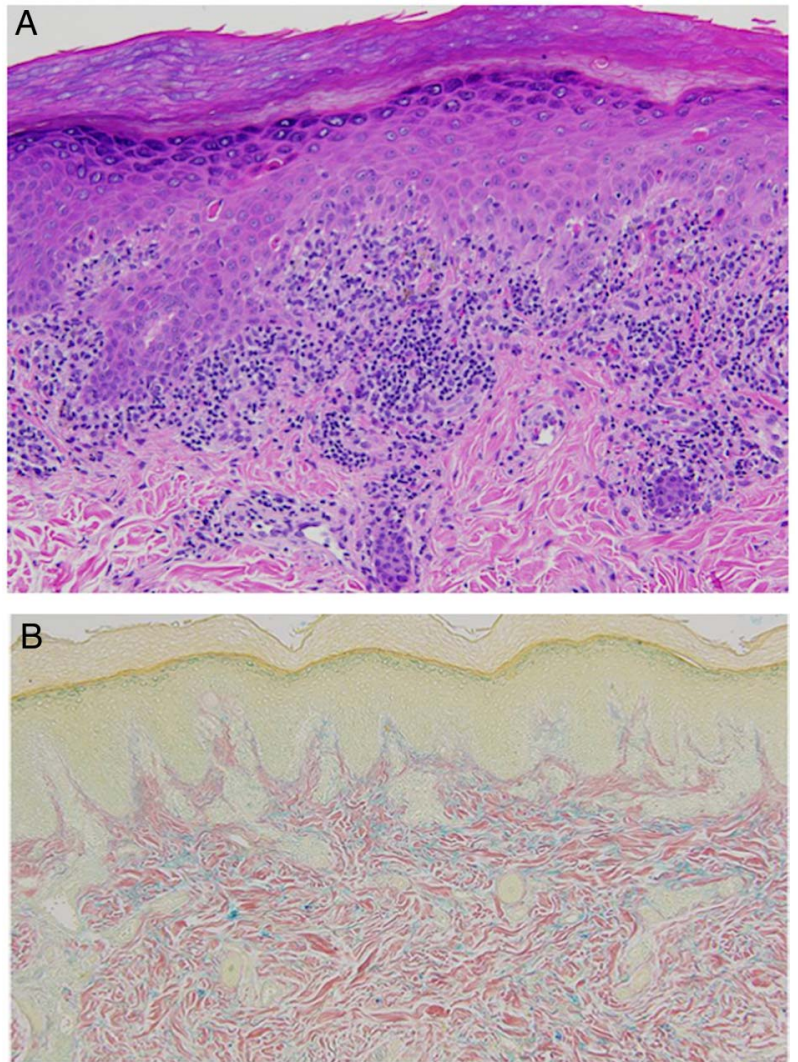

Figure 2 (A) Topical terbinafine-induced subacute cutaneous lupus erythematosus (SCLE). H\&E stain revealed hyperkeratosis, superficial and deep perivascular lymphohistiocytic infiltrate, vacuolar changes and deposits of connective tissue mucin in dermis. (B) Topical terbinafine-induced SCLE. Colloidal iron stain revealed increased deposits of connective tissue mucin throughout the dermis, most prominent in the reticular dermis.

drug-induced SCLE were older, had more extensive skin involvement and had an increased incidence of bullous, erythema multiforme-like and vasculitic lesions compared with individuals with idiopathic SCLE. ${ }^{7}$ Photodistribution is characteristic as in our patient, and resolution occurred within a few months without treatment as noted in the literature. ${ }^{8}$ Interestingly, our patient's unusual presentation of localised SCLE lesions on the hands may be explained by an occupational exposure to a topical drug, namely terbinafine.

As a foot masseuse, the patient was exposed almost daily to topical terbinafine. Systemic administration of this agent is a well-known cause of drug-induced SCLE, but there are no reports of topical induction of SCLE. ${ }^{1} 689$ Terbinafine is an allylamine with antimycotic effects that is used in the treatment of fungal foot and nail infections. ${ }^{10}$ It is a highly lipophilic substance that may concentrate in the skin, nails and fatty tissue for a prolonged period of time ${ }^{10}$ While topical terbinafine is not readily absorbed into the systemic circulation and therefore would unlikely cause systemic lupus flare, the possibility arises that if locally absorbed it may induce focal development of $\operatorname{SCLE}^{10}$ Studies have 
demonstrated that the average incubation time for terbinafine-induced SCLE was 5.1 weeks (range 1-12 weeks). ${ }^{6}{ }^{9}$ Our patient's initial lesions on the dorsal hand and interdigital spaces may have resulted from prolonged exposure to topical terbinafine.

Our patient's cutaneous manifestation of SCLE on the hands is noteworthy because of the sparing of the palmar surfaces. In an occupational exposure as a foot masseuse, one would expect lesions on the volar surfaces as well. One possible explanation is that the thickness of the palmar surface of the hand limited the permeability of topical terbinafine, as opposed to the thinner skin on the dorsum of the hand. Studies have shown that skin permeability is variable in different areas of the body, being generally greatest in thin-skinned surfaces. ${ }^{11}$ Another explanation for involvement of the dorsum of the hand versus the palmar surface is increased exposure to ambient Ultraviolet (UV) radiation. Whether the UV radiation, medication or a combination of these exogenous factors precipitated the patient's condition is not clear.

There are suggested hypotheses for the pathogenesis of idiopathic and drug-induced SCLE. Genetics have been shown to play an important role in the development of SCLE, specifically in individuals with human leucocyte antigen (HLA) B8, HLA-DR3, HLA-DRw52 and HLA-DQ1. ${ }^{12}$ It is speculated that UV radiation stimulates keratinocytes and dermal fibroblasts to release cytokines such as TNF- $\alpha$ and interleukins involved in immunomodulation and apoptotic pathways in SCLE. ${ }^{13}$ In addition, nuclear antigens (including anti-SSA/Ro) in keratinocytes that have been translocated to the cell surface may be targeted by circulating anti-SSA/Ro antibodies and cytotoxic T cells. ${ }^{73}$ In drug-induced SCLE, the induction of a photosensitivity state by certain medications may induce skin lesions via an isomorphic (Köebner) response in predisposed individuals. ${ }^{2}$ Terbinafine is capable of producing a photosensitivity state and is speculated to induce SCLE through this mechanism. ${ }^{2}$ The pathogenesis of drug-induced SCLE may vary according to individual medications. ${ }^{2}$ For example, hydrochlorothiazide is hypothesised to enhance the cytotoxicity of anti-Ro antibodies and induce epidermal cytotoxicity through direct phototoxicity. ${ }^{3}$

This case represents a rare and interesting instance of SCLE isolated to the dorsum of the hand and interdigital spaces. We propose that localised exposure of the hands to topical terbinafine in an occupational setting was the cause of this unusual presentation. Although little is known about the absorption and pharmacodynamics of topical terbinafine, its potential to induce SCLE topically deserves further investigation.
Contributors JSL, LGF, AM, SMR, RFW and AGF had full access to all of the data in the study and take responsibility for the integrity of the data and the accuracy of the data analysis. Study concept and design: SMR and AGF. Acquisition, analysis and interpretation of data: JSL, AGF, AM, SMR, RFW and AGF. Drafting of the manuscript: JSL, LGF, SMR and AGF. Critical revision of the manuscript for important intellectual content: SMR and AGF. Obtained funding: AGF. Study supervision: AGF.

Funding The Frances and Benjamin Benenson Foundation and Lynn and William M. Silverman Donation Fund.

Competing interests None declared.

Provenance and peer review Not commissioned; externally peer reviewed.

Data sharing statement No additional data are available.

Open Access This is an Open Access article distributed in accordance with the Creative Commons Attribution Non Commercial (CC BY-NC 4.0) license, which permits others to distribute, remix, adapt, build upon this work noncommercially, and license their derivative works on different terms, provided the original work is properly cited and the use is non-commercial. See: http:// creativecommons.org/licenses/by-nc/4.0/

\section{REFERENCES}

1. Werth VP. Clinical manifestations of cutaneous lupus erythematosus. Autoimmun Rev 2005;4:296-302.

2. Lowe GC, Henderson $\mathrm{CL}$, Grau $\mathrm{RH}$, et al. A systematic review of drug-induced subacute cutaneous lupus erythematosus. Br J Dermatol 2011;164:465-72.

3. Shapiro M, Sosis AC, Junkins-Hopkins JM, et al. Lupus erythematosus induced by medications, ultraviolet radiation, and other exogenous agents: a review, with special focus on the development of subacute cutaneous lupus erythematosus in a genetically predisposed individual. Int $J$ Dermatol 2004;43:87-94.

4. Ashinoff R, Werth VP, Franks AG, Jr. Resistant discoid lupus erythematosus of palms and soles: successful treatment with azathioprine. J Am Acad Dermatol 1988;19:961.

5. Prystowsky SD, Herndon JH, Jr, Gilliam JN. Chronic cutaneous lupus erythematosus (DLE) - a clinical and laboratory investigation of 80 patients. Medicine (Baltimore) 1976;55:183-91.

6. Lorentz K, Booken N, Goerdt S, et al. Subacute cutaneous lupus erythematosus induced by terbinafine: case report and review of literature. J Dtsch Dermatol Ges 2008;6:823-7, 823-8.

7. Marzano AV, Lazzari R, Polloni I, et al. Drug-induced subacute cutaneous lupus erythematosus: evidence for differences from its idiopathic counterpart. Br J Dermatol 2011;165:335-41.

8. Srivastava M, Rencic A, Diglio G, et al. Drug-induced, Ro/ SSA-positive cutaneous lupus erythematosus. Arch Dermatol 2003;139:45-9.

9. Kasperkiewicz M, Anemüller W, Angelova-Fischer I, et al. Subacute cutaneous lupus erythematosus associated with terbinafine. Clin Exp Dermatol 2009;34:e403-4.

10. Hill S, Thomas R, Smith SG, et al. An investigation of the pharmacokinetics of topical terbinafine (Lamisil) $1 \%$ cream. Br J Dermatol 1992;127:396-400.

11. Cronin E, Stoughton RB. Percutaneous absorption. Regional variations and the effect of hydration and epidermal stripping. Br J Dermatol 1962;74:265-72.

12. Lin JH, Dutz JP, Sontheimer RD, et al. Pathophysiology of cutaneous lupus erythematosus. Clin Rev Allergy Immunol 2007;33:85-106.

13. Werth VP, Zhang W, Dortzbach K, et al. Association of a promoter polymorphism of tumor necrosis factor-alpha with subacute cutaneous lupus erythematosus and distinct photoregulation of transcription. J Invest Dermatol 2000;115:726-30. 